\title{
Otologic Findings Based on no Complaints in a Pediatric Examination
}

\author{
Emine Demir $^{1}$ Sevgi Topal ${ }^{2} \quad$ Gorkem Atsal $^{3} \quad$ Mehmet Erdil $^{4}$ Zerrin Ozergin Coskun ${ }^{1}$ Engin Dursun ${ }^{1}$ \\ ${ }^{1}$ Department of Otorhinolaryngology Head and Neck Surgery, \\ Address for correspondence Emine Demir, Department of \\ Faculty of Medicine, Recep Tayyip Erdogan University, Rize, Turkey \\ ${ }^{2}$ Department of Pediatrics, Agri State Hospital, Agri, Turkey \\ ${ }^{3}$ Department of Otorhinolaryngology Head and Neck Surgery, \\ Otorhinolaryngology Head and Neck Surgery, Faculty of Medicine, \\ Recep Tayyip Erdogan University Training and Research Hospital, \\ ENT Clinic, Rize, Turkey (e-mail: emine.demir@erdogan.edu.tr).
}

Izmir Bozyaka Training and Research Hospital, Izmir, Turkey

${ }^{4}$ Department of Otorhinolaryngology Head and Neck Surgery,

Agri State Hospital, Agri, Turkey

Int Arch Otorhinolaryngol 2019;23:36-40.

\begin{abstract}
Keywords

- otoscopy

- pediatrics

- otologic

Introduction the otological evaluation is highly significant in those branches of medicine in which the general evaluation of the patient is conducted by general practitioners and pediatricians.

Objectives To investigate the otologic findings and their incidences in the sample, which consisted of patients who presented to the pediatric outpatient clinic with nonotologic complaints.

Methods Patients, aged between 2 and 16 years, who had neither otorhinolaryngological complaints nor history of surgery by the otolaryngology (ear, nose and throat, ENT) department were included in the present study. The findings detected in the external auditory system and in the tympanic membranes as well as the otorhinolaryngologists' otologic examination findings of the referred patients were recorded from the files of each patient along with the applied medical and surgical treatments, and diagnostic investigations.

Results Of a total of 973 patients evaluated, 129 (13.2\%) were referred to the ENT outpatient clinic due to any otologic pathology. In the otorhinolaryngological examinations, false positivity was detected in $12(1.2 \%)$ patients, and pathological findings were detected in 117 (12\%) patients who received the following diagnoses: 68 (6.9\%) had otitis media with effusion(OME); $37(3.8 \%)$ had cerumen impactions; $8(0.8 \%)$ had acute otitis media (AOM); $2(0.2 \%)$ had ticks in the external auditory canal; $2(0.2 \%)$ had a retraction pocket in tympanic membrane; $1(0.1 \%)$ had unilateral central dry perforation of the tympanic membrane; and $1(0.1 \%)$ had congenital cholesteatoma. Conclusion Any complications and sequelae that may develop due to any pathologies and predominantly infections can be prevented by an early diagnosis, which can be made by means of a simple examination, performed merely with an otoscope. Pediatricians and general practitioners should be in close contact with otorhinolaryngologists regarding the pathologies they identify.
\end{abstract}

received

January 24, 2018

accepted

May 23, 2018

published online

July 25,2018
DOI https://doi.org/

10.1055/s-0038-1667007. ISSN 1809-9777.
Copyright (e) 2019 by Thieme Revinter

Publicações Ltda, Rio de Janeiro, Brazil
License terms

(c) (i) $\ominus$ (\$) 


\section{Introduction}

The otologic evaluation is highly significant in those branches of medicine in which the general evaluation of the patient is conducted by general practitioners and pediatricians. The otoscopic examination should become a part of the routine examination. An early diagnosis of primary infections and of any pathology during a simple examination using only an otoscope can prevent possible complications and sequelae. Besides, misdiagnoses determined by pediatricians are a clinical problem that may result in unnecessary treatment. ${ }^{1,2}$ Therefore, pediatricians must be in close interaction with otorhinolaryngologists regarding the methodology of the detected pathologies. Hence, it will be more convenient to manage diseases through a correct diagnosis and treatment. In addition, the branches of medicine in which the general evaluation of the patient is performed will gain experience, the percentages of correct diagnoses will rise, and unnecessary treatments will be avoided.

\section{Objective}

The aim of the present study is to assess otologic findings in pediatric patients without any ENT complaints who presented to a polyclinic for any reason.

\section{Methods}

After receiving the approval from Ethics Committee (protocol number 215), the authors signed the informed consent form and indicated that there were no conflicts of interest in the present study.

A total of 973 patients were included in the study. The ages of the patients ranged from 2 to 16 years. The patients presented to a pediatric polyclinic between June 2016 and June 2017 for any reason; however, they did not have any otorhinolaryngological complaints, and no history of otorhi- nolaryngological surgery. All patients were evaluated by the same pediatrician. Findings regarding the external auditory canal (EAC) and the tympanic membrane obtained during the otoscopic examination were registered from the file of each patient. Patients with any detected pathology were referred to an otorhinolaryngologist. The otoscopic findings, medical, and surgical treatments and the examinations for the diagnosis of those patients were recorded.

Patients with otitis media with effusion (OME) were submitted to a tympanogram. The measurements of the tympanometry were evaluated as type A, B and C, according to the Jerger classification system. ${ }^{3}$

\section{Results}

Of a total of 973 patients included in the present study, 556 (58.2\%) were male, and 407 (41.8\%) were female. The age of the patients ranged from 2 to 16 years, and the mean age was 8.9 years. The results of the otoscopic examination of 844 (86.8\%) of the patients were normal. A total of 129 (13.2\%) of them were referred to the otorhinolaryngology department due to an ear pathology ( $\mathbf{- F i g . ~ 1 ) . ~ O u t ~ o f ~ t h e ~ t o t a l ~ o f ~} 844$ referred patients, 12 were observed with myringosclerosis and atrophic membrane indistinguishable from perforation, which represents a false positivity rate of $1.2 \%$ in the pediatrician's examination. Pathologic findings were detected in the otoscopic examinations of 117 (12\%) patients in the post otorhinolaryngological examination (-Fig. 2).

A total of 117 patients diagnosed with pathologies were evaluated. A total of 68 (6.9\%) patients were diagnosed with OME, which was unilateral in 14 (20.5\%) patients and bilateral in 54 (79.5\%) patients. Regarding the evaluation of the tympanograms, type B was detected in 51 (75\%) patients, while type $C$ was detected in 17 (25\%) patients. Cerumen impaction (25 bilateral, 12 unilateral) was detected in 37 (3.8\%) patients. Acute otitis media (AOM) was diagnosed in $8(0.8 \%)$ patients ( 5 unilateral, 3 bilateral). One of the patients diagnosed with

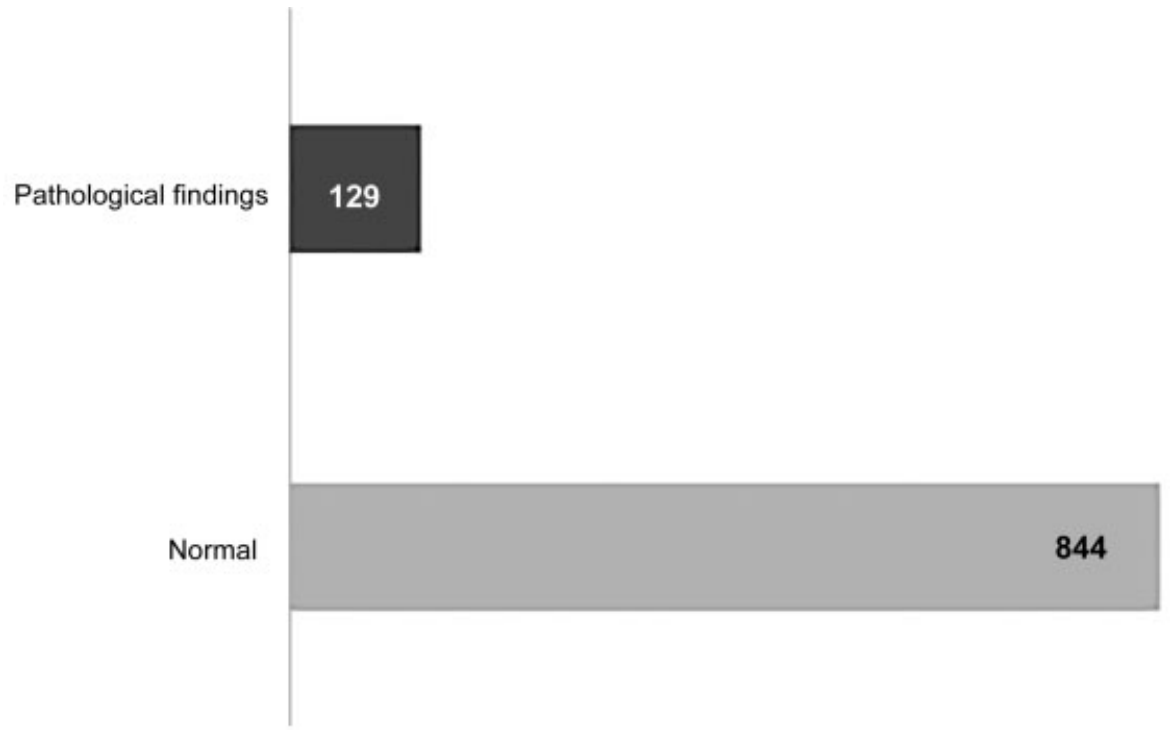

Fig. 1 Pediatrician's otoscopic examination findings. 


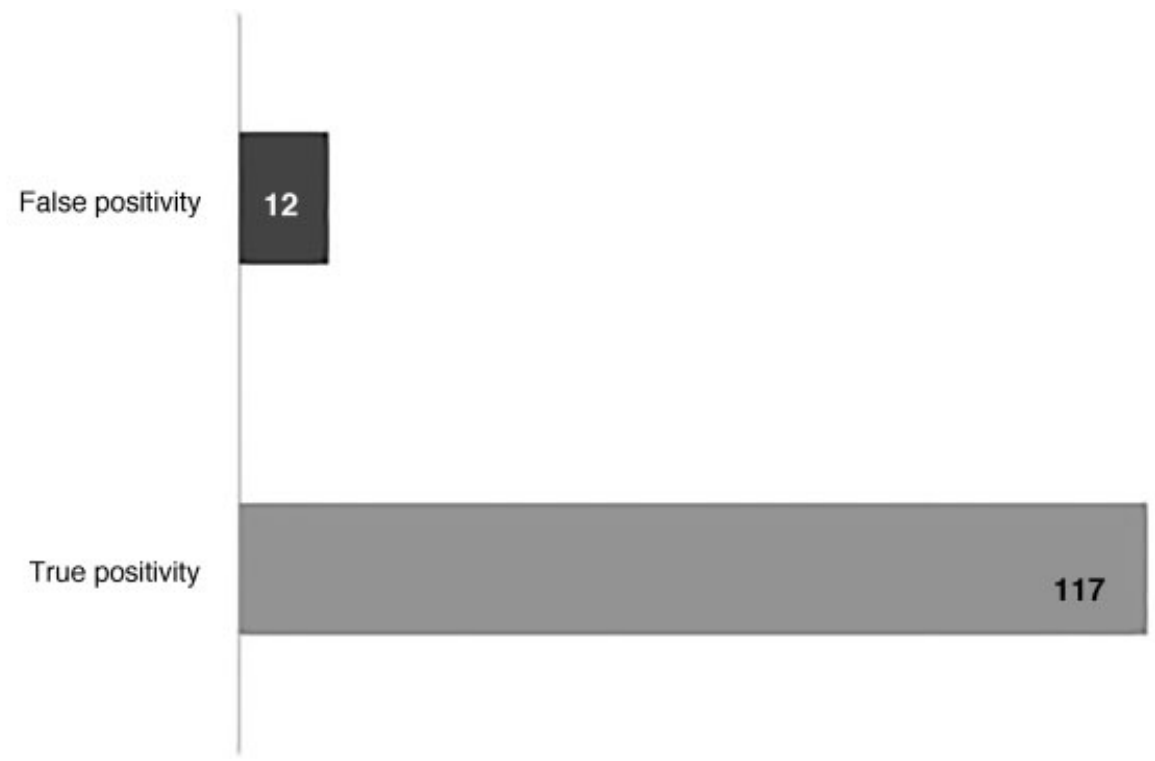

Fig. 2 Patient's otoscopic findings, with true or false positivity numbers of patients who were referred from a pediatrician to an otorhinolaryngologist.

AOM presented the following complaints: nausea, vomiting and discomfort while seven of them had fever. A foreign body (tick) was detected in the EACs of $2(0.2 \%)$ patients. Unilateral central dry perforation in the tympanic membrane was observed in $1(0.1 \%)$ patient, while retraction pockets were observed in $2(0.2 \%)$ patients. A type C tympanogram was obtained as a result of the tympanometry test performed on the patients with retraction pockets. Congenital cholesteatoma was prediagnosed in $1(0.1 / \%)$ patient whose computed tomography of the temporal bone (CTTB) and diffusion magnetic resonance imaging (MRI) tests were conducted by the otorhinolaryngologist; the patient was referred to surgery, and the final diagnosis of cholesteatoma was determined as a result of the histopathological evaluation of the specimen ( - Table 1 ).

\section{Discussion}

Additional otologic findings have been obtained, which may be significant to be diagnosed or noticed in a rate of $12 \%$ in the

Table 1 All patients' pathological findings in the routine examination and rates compared to all examined patients

\begin{tabular}{|l|l|}
\hline $\begin{array}{l}\text { Pathological findings in } \\
\text { the routine examination }\end{array}$ & Rates \\
\hline OME & $68(6.9 \%)$ \\
\hline Cerumen impaction & $37(3.8 \%)$ \\
\hline AOM & $8(0.8 \%)$ \\
\hline Foreign body (tick) in the EAC & $2(0.2 \%)$ \\
\hline Retraction pocket in tympanic membrane & $2(0.2 \%)$ \\
\hline Perforation of the tympanic membrane & $1(0.1 \%)$ \\
\hline Congenital cholesteatoma & $1(0.1 \%)$ \\
\hline
\end{tabular}

Abbreviations: AOM, acute otitis media; EAC, external auditory canal; OME, otitis media with effusion. pediatric patient group that presented to the pediatrics department without any otorhinolaryngological complaints, but with various other complaints. The findings detected during the examination were OME, cerumen impaction, AOM, and live foreign body (tick) in the EAC. Pathologies such as congenital cholesteatoma and tympanic membrane perforation or retraction are vital issues both for a child's healthcare and for his/her speech and language development.

Otitis media with effusion is an accumulation of fluid in the middle ear without acute infection symptoms and findings. Ninety percent of the children experience at least once OME attack before school age, which is the most common reason for hearing loss in children. ${ }^{4}$ The prevalence of OME has been found to be between $10 \%$ to $40 \%$ in several studies. ${ }^{5-7}$ However, in the present study, it was found in $6.9 \%$ of the cases. However, none of these patients had any complaints about their ears, which we think is the reason why we found a lower result. It is significant to diagnose OME and perform a follow-up of the patients in order to prevent possible complications. Chronic otitis media, adhesive otitis media, formation of a retraction pocket and tympanosclerosis are common complications. If not treated regularly, they may cause permanent hearing loss. ${ }^{8,9}$ In addition, OME not treated for a long period has such risks as language and speech disorders and learning difficulties due to hearing loss in children. ${ }^{7,9}$ It is significant that pediatric patients should be evaluated by pediatricians for these reasons. Furthermore, it is essential to receive a reliable medical history from the caretaker, and to inform him/her about the disease and the possible sequelae.

Cerumen is secreted by the EAC for protection, lubrication and cleaning. Cerumen is removed from the EAC through a self-cleaning function. However, the accumulation of cerumen in the EAC causes cerumen impaction. ${ }^{10}$ Cerumen impaction may result in hearing difficulty, pain and loss of balance. Sneaky hearing loss caused by cerumen impaction 
may bring about serious problems in children during a critical period for speech and language development. ${ }^{11}$ As it has been observed in the present study, neither a caretaker nor a child may recognize cerumen impaction and its symptoms. We think the percentage of asymptomatic cerumen impaction is high in the present study. This may result from the fact that we have carried out the study in a region where the sociocultural level is low. However, although it seems insignificant, the detection of cerumen impaction during routine examination has serious impacts on a child's speech and language development and success at school.

Acute otitis media is an inflammation of the middle ear accompanied by systemic infection findings. Earache and fever are the most encountered symptoms. However, in the hyperemia phase, which is the beginning phase of AOM, the earache has not started yet, and the fever is at a low grade. In this phase, AOM is usually diagnosed circumstantially. ${ }^{12}$ Sometimes the hyperemia formed in the tympanic membrane, especially while a baby is crying, may result in misdiagnosis. An accurate diagnosis of AOM is significant to remove symptoms of the infection and prevent possible complications, while the detection of a misdiagnosis is important to avoid the unnecessary use of antibiotics. ${ }^{13,14}$ All of the patients referred to us with suspicion of AOM were evaluated and diagnosed on the same day. Only one of the patients had middle ear exudate and others had only hyperemia. The patients were at the beginning phase of AOM, and only had symptoms of systemic infection. Their medical treatments were arranged and the disease was controlled without progression. It is easy to manage the disease and avoid complications thanks to an early diagnosis.

Ticks mediate as a vectors that transfer infections from various microorganisms to humans. ${ }^{15}$ They infect humans through animal or environmental contact in rural regions. Ticks attaching inside the EAC may cause serious systematic effects, which may result in death, unlike other foreign bodies. They may bring about various symptoms, such as earache, abrasion in the skin of the EAC, sensation of ear fullness and local infection. ${ }^{16}$ Sometimes, they do not present any symptoms. ${ }^{17}$ Ticks were encountered in two patients during the routine ear examination without causing any symptoms. We may have encountered ticks because the region where the study was performed is rural. The patients were 11 and 13 years old. The ticks were removed with alligator forceps without any need for general anesthesia. An infection consultation was performed for both patients and they underwent a close follow-up. The follow-ups of the patients who did not present with any symptoms such as fever or muscle pain and who did not develop thrombocytopenia, leucopoenia and coagulation disorder in the laboratory assessments were finished one week later. It is critical to diagnose these patients considering both the systematic and ontological effects the ticks may cause even if they are asymptomatic.

Congenital cholesteatoma is seen as a white mass behind a normal intact tympanic membrane. ${ }^{18}$ It is difficult to diagnose, since it is asymptomatic in the early phase. ${ }^{19}$ It may cause irreversible damages both in hearing and in the temporal bone if the diagnosis is delayed. The fact that many children are asymptomatic and otoscopic findings are obscure makes it difficult for the doctor to diagnose. ${ }^{20}$ The diagnosis of the 3-year-old boy in our study was performed by the pediatrician through an otoscopy. The pathological results of the patient confirmed the diagnosis. When there is a suspicion of congenital cholesteatoma, a careful otoscopic examination may bring about the diagnosis.

The tympanic membrane helps the transmission of sound to the ossicles by means of vibration. ${ }^{21}$ If the tympanic membrane is perforated, the surface area where this transmission is allowed will be reduced. Therefore, hearing will be impacted. $^{22}$ Its retraction, which is defined as tympanic membrane retracted to middle ear may also impact the hearing function. If untreated, it can cause perforation of the tympanic membrane, damage in the ossicular chain, and cholesteatoma. ${ }^{23}$ Both perforation of the tympanic membrane and its retraction can have serious effects on hearing and, consequently, on the mental development of children. To this end, patients must be monitored closely through hearing tests and otoscopic examinations in case they need to be submitted to surgical treatment.

In the literature review, we have not encountered any studies providing otologic findings of patients without such otorhinolaryngological complaints. Only Kocyigit et $\mathrm{al}^{7}$ researched the incidence of OME. We have evaluated all otologic findings in the present study. The limitation of the present study can be the fact that it has been designed for children and only to evaluate otologic findings. More comprehensive data can be obtained by evaluating total ENT conditions in broader patient scales. We think different results can be achieved from studies in which a higher number of physicians and broader patient scales are included in multiple centers. In the present study, the rate of false positive diagnosis by the pediatrician was of $1.2 \%$. But one of the limitations of the study was the inability to calculate false negative diagnostic rates. Studies in which all patients are assessed by both a pediatrician and an otorhinolaryngologist can be designed.

\section{Conclusion}

Family doctors, pediatricians and internal medicine specialists performing overall evaluations of patients should not ignore the ENT examination. As seen in the present study, pathologies that are likely to result in sequela in the future may be diagnosed through simple examination methods. Early diagnosis and treatment may prevent these sequelae. The otoscopic examination is even adequate when there are suspicions of ailments. Advanced diagnosis and treatment may avoid the formation of sequelae.

\section{References}

1 Lundberg T, Biagio de Jager L, Swanepoel W, Laurent C. Diagnostic accuracy of a general practitioner with video-otoscopy collected by a health care facilitator compared to traditional otoscopy. Int J Pediatr Otorhinolaryngol 2017;99(02):49-53 
2 Lieberthal AS, Carroll AE, Chonmaitree T, et al. The diagnosis and management of acute otitis media. Pediatrics 2013;131(03): e964-e999

3 Jerger J. Clinical experience with impedance audiometry. Arch Otolaryngol 1970;92(04):311-324

4 Tos M. Epidemiology and natural history of secretory otitis. Am J Otol 1984;5(06):459-462

5 Sanli A, Tasdemir O, Eken M, Celebi O, Yilmaz SH. Prevalence of otitis media with effusion among primary school age-children and etiopathogenic examination. Indian J Otolaryngol Head Neck Surg 2014;66(Suppl 1):95-98

6 Paradise JL, Rockette HE, Colborn DK, et al. Otitis media in 2253 Pittsburgh-area infants: prevalence and risk factors during the first two years of life. Pediatrics 1997;99(03):318-333

7 Kocyigit M, Ortekin SG, Cakabay T, Ozkaya G, Bezgin SU, Adali MK. Frequency of Serous Otitis Media in Children without Otolaryngological Symptoms. Int Arch Otorhinolaryngol 2017;21(02): 161-164

8 Coyte PC, Croxford R, Asche CV, To T, Feldman W, Friedberg J. Physician and population determinants of rates of middle-ear surgery in Ontario. JAMA 2001;286(17):2128-2135

9 Ito $\mathrm{M}$, Takahashi $\mathrm{H}$, Iino $\mathrm{Y}$, et al. Clinical practice guidelines for the diagnosis and management of otitis media with effusion (OME) in children in Japan, 2015. Auris Nasus Larynx 2017;44(05):501-508

10 Mandour MA, El-Ghazzawi EF, Toppozada HH, Malaty HA. Histological and histochemical study of the activity of ceruminous glands in normal and excessive wax accumulation. J Laryngol Otol 1974;88(11):1075-1085

11 Ping C, Yanling H, Youhua W, Shufen W, Zhinan W, Zhongfang X. Epidemiology of cerumen impaction among municipal kindergartens children in Wuhan, China. Int J Pediatr Otorhinolaryngol 2017;100(02):154-156

12 Pichichero ME. Otitis media. Pediatr Clin North Am 2013;60(02): 391-407
13 Isaacson G. Otoscopic diagnosis of otitis media. Minerva Pediatr 2016;68(06):470-477

14 Varsak YK, Gül Z, Eryılmaz MA, Arbağ H. Prevalence of otitis media with effusion among school age children in rural parts of Konya province, Turkey. Kulak Burun Bogaz Ihtis Derg 2015;25(04): 200-204

15 Bursali A, Keskin A, Tekin S. A review of the ticks (Acari: Ixodida) of Turkey: species diversity, hosts and geographical distribution. Exp Appl Acarol 2012;57(01):91-104

16 Gökdoğan O, Çakabay T, Baran H, Karabulut B, Tasdemir C, Vatansever Z. Otoacariasis: demographic and clinical outcomes of patients with ticks in the ear canal. Rev Bras Otorrinolaringol (Engl Ed) 2016;82(04):416-421

17 Erenler AK, Kulaksiz F, Ülger H, et al. Characteristics of patients admitted to the emergency department due to tick bite. Trop Doct 2014;44(02):86-88

18 Kojima H, Tanaka Y, Shiwa M, Sakurai Y, Moriyama H. Congenital cholesteatoma clinical features and surgical results. Am J Otolaryngol 2006;27(05):299-305

19 Cho HS, Kim HG, Jung DJ, Jang JH, Lee SH, Lee KY. Clinical Aspects and Surgical Outcomes of Congenital Cholesteatoma in 93 Children: Increasing Trends of Congenital Cholesteatoma from 1997 through 2012. J Audiol Otol 2016;20(03):168-173

20 Walker D, Shinners MJ. Congenital Cholesteatoma. Pediatr Ann 2016;45(05):e167-e170

21 Voss SE, Rosowski JJ, Merchant SN, Peake WT. How do tympanicmembrane perforations affect human middle-ear sound transmission? Acta Otolaryngol 2001;121(02):169-173

22 Dawood MR. Frequency Dependence Hearing Loss Evaluation in Perforated Tympanic Membrane. Int Arch Otorhinolaryngol 2017; 21(04):336-342

23 James AL, Papsin BC, Trimble K, et al. Tympanic membrane retraction: An endoscopic evaluation of staging systems. Laryngoscope 2012;122(05):1115-1120 